\title{
Comparación de tres conceptos afines de homonegatividad entre tres facultades de ciencias de la salud
}

\section{Comparison of three related concepts of homonegativity among three faculties of health sciences}

José Moral de la Rubia

Universidad Autónoma de Nuevo León

\author{
Adrián Valle de la $\mathrm{O}$ \\ Instituto Tecnológico y de Estudios \\ Superiores de Monterrey
}

\section{Resumen}

El presente estudio tiene como objetivos: 1) definir dos componentes de rechazo hacia personas no heterosexuales (abierto/externo y sutil/interno); 2) calcular las medias de rechazo, 3) comparar las medias de rechazo entre tres facultades de ciencias de la salud y 4) controlar el efecto del sexo en estas diferencias. Se aplicaron las escalas de Actitudes hacia Lesbianas y Hombres Homosexuales (ATLG), Homonegatividad Internalizada (HNI-16) y Homofobia (HF-8) a una muestra no probabilística de 231 estudiantes (54\% mujeres y $48 \%$ hombres). De los 231 participantes, 100 fueron encuestados en una facultad pública de medicina, 66 en una facultad privada de medicina y 65 en una facultad pública de psicología. Se definieron los componentes esperados de rechazo abierto/externo y sutil/interno. Las medias de las subescalas y el componente de rechazo abierto/externo correspondieron a una respuesta de desacuerdo con el rechazo. Las medias de las subescalas y el componente de rechazo sutil/interno correspondieron a una respuesta de ambigüedad entre la aceptación y el rechazo. Las medias entre las tres facultades fueron estadísticamente diferenciales en las subescalas de rechazo sutil hacia los hombres homosexuales (ATG-S), rechazo de la manifestación pública de la homosexualidad (EXT) y en el componente de rechazo sutil/interno. La subescala de rechazo de fantasías, deseos e identidad homosexuales propios (INT) fue diferencial, cuando el sexo fue parcializado. Los tamaños de efectos fueron pequeños. Se concluyó que la variable carrera tuvo un efecto significativo sobre el rechazo hacia las personas no heterosexuales. La aceptación fue mayor entre estudiantes de psicología que de medicina.

Palabras clave: diversidad sexual, actitud, homonegatividad, homofobia, estudiantes.

Nota del autor

José Moral de la Rubia, Facultad de Psicología, Universidad Autónoma de Nuevo León; Adrián Valle de la O, Departamento de Ciencias Básicas de la Escuela de Medicina y Ciencias de la Salud, Instituto Tecnológico y de Estudios Superiores de Monterrey (ITESM).

La correspondencia en relación con este artículo debe dirigirse a José Moral de la Rubia, Facultad de Psicología, Universidad Autónoma de Nuevo León, c/Dr. Darlos Canseco 110, col. Mitras Centro, C.P. 64460, Monterrey, Nuevo León, México.

Dirección electrónica: jose_moral@hotmail.com 


\begin{abstract}
The aims of this study were to: 1) define two components of (open/external and subtle/internal) rejection towards non-heterosexual persons; 2) calculate the means of rejection; 3) compare the means of rejection among three schools of health sciences and 4) controlling the effect of sex in these differences. The Attitudes towards Lesbians and Gay Men (ATLG) scale, the 16-item Internalized Homonegativity (IHN-16) scale and the 8-item Homophobia (HF-8) scale were applied to a non-probability sample of 231 participants (54\% women and $46 \%$ men). One hundred students were surveyed in a public school of medicine, 66 in a private school of medicine and 65 in a public school of psychology. The two expected components were defined, one of open/external rejection and another of subtle/internal rejection. The means of the open/external rejection subscales and component corresponded to an answer of disagreement with the rejection. The means of the subtle/internal rejection subscales and component corresponded to an answer of ambiguity between rejection and acceptance. The means among the three schools were statistically differential in the subscales of subtle rejection towards homosexual men (ATG-S) and rejection towards public manifestation of homosexuality (EXT), and in the subtle/internal rejection component. The means of the rejection towards own homosexual fantasies, desires and identity (INT) subscale were differential among the three schools when sex was partialized. Effect sizes were small. It is concluded that the variable career had a significant effect on the rejection towards non-heterosexual persons. Acceptance was greater among psychology students than among medicine students.
\end{abstract}

Keywords: sexual diversity, attitude, homonegativity, homophobia, students.

Las minorías sexuales comprenden muy diversos grupos que históricamente han recibido una atención inadecuada, si no discriminatoria, en los países desarrollados; dicha situación es más acentuada en países en vías de desarrollo. Sin embargo, en la mayoría de los países, los profesionales de la salud no reciben la formación específica sobre los problemas que enfrentan estos grupos, por consiguiente, se registra una falta de centros de atención clínica amigables para estas personas (Mayer et al., 2012).

En gran parte de los currículos de los estudiantes de ciencias de la salud no se aborda de manera amplia y clara el tópico de la diversidad sexual. El desarrollo de un plan de estudios en el cual se aborden las necesidades en salud de las personas no heterosexuales es un primer paso para hacer frente a los prejuicios, a menudo encubiertos, en los futuros profesionales en el campo del cuidado de la salud. El empleo de métodos educativos más interactivos y que integren diversos campos de estudio, como antropología, sociología, literatura, etc., puede ser de gran utilidad para brindar a los estudiantes foros diversos donde exploren y discutan sus propias creencias y actitudes sobre la diversidad humana y la multiplicidad de estilos de vida, para promover así el surgimiento de empatía no sólo hacia las minorías sexuales, sino también hacia personas de diversos orígenes étnicos y sociales (Matharu et al., 2012; Selleger, Bonke, \& Leeman, 2006). 
Dentro de la investigación social del rechazo hacia las personas no heterosexuales se distinguen tres conceptos afines: actitud, homofobia y homonegatividad. El concepto de actitud hace referencia al posicionamiento valorativo de un individuo frente a un objeto (Haddock, 2004). En la actitud hacia las personas no heterosexuales se distingue un aspecto de rechazo abierto con conductas agresivas $y$ discriminación manifiesta frente a un aspecto de rechazo sutil o simbólico con un trato diferencial y matizaciones devaluadoras subrepticias (Herek, 2004; Morrison, Speakman, \& Ryan, 2009). El rechazo abierto implica una intención deliberada o consciente y es afín a la actitud explícita. El rechazo sutil o simbólico usualmente aparece como una forma automática de reacción ante la presencia del objeto actitudinal y está más relacionado con el concepto de actitud implícita (Cárdenas \& Barrientos, 2008).

La homofobia hace referencia a una actitud rígida de rechazo con ideas estereotipadas y conductas discriminatorias y agresivas (Barrientos \& Cárdenas, 2013; Herek, 2004). Cuando la homofobia es evaluada en individuos con orientación heterosexual (rechazo dirigido al otro distinto de mí), se habla de homofobia externalizada; cuando es evaluada en individuos con conductas sexuales con personas de su mismo sexo (rechazo dirigido hacia sí mismo y los otros como yo), se habla de homofobia internalizada (Herek, Gillis, \& Cogan, 2009). El término ha recibido críticas por su connotación psicopatológica (fobia específica) y por oscurecer el origen sociocultural del rechazo visceral hacia las personas no heterosexuales (Herek \& McLemore, 2013), de ahí que se haya introducido el de homonegatividad para sustituirlo.

La homonegatividad hace referencia a las creencias prejuiciosas, reacciones emocionales negativas, actitud de rechazo y conductas de estigmatización y discriminación hacia las personas no heterosexuales. También se distingue una homonegatividad externalizada, dirigida contra el otro distinto de mí en personas exclusivamente heterosexuales, de una homonegatividad internalizada, dirigida contra el deseo propio hacia personas del mismo sexo y la manifestación pública de la diversidad sexual en personas no heterosexuales (Currie, Cunningham, \& Findlay, 2004).

En el presente estudio, con base en la escala de Homonegatividad Internalizada (HNI-16; Moral \& Valle, 2013a), se da un sentido más amplio a la noción de homonegatividad internalizada, al considerar que el concepto puede ser aplicado a cualquier individuo de forma independiente de la orientación sexual autodefinida, debido a que las fantasías y deseos hacia personas del mismo sexo pueden manifestarse en cualquier persona (bisexualidad potencial) y que cualquier individuo puede experimentar miedo a revelar estos sentimientos $\mathrm{y} / \mathrm{o}$ mostrar conductas desviadas de su rol de género. Desde esta perspectiva, el concepto pone el acento en las vivencias internas (rechazo hacia sí mismo) y en la mirada prejuiciosa o condenatoria del otro internalizada, dentro de una sociedad con valores 
heterosexistas en la cual persiste un rechazo sutil hacia las personas no heterosexuales (Moral \& Valle, 2013a; Szymanski, 2008).

Dentro de la sociedad occidental, tradicionalmente las personas no heterosexuales han sido estigmatizadas y discriminadas. A partir de la década de 1970, esta actitud de condena abierta ha tendido a desaparecer y ser sustituida por una actitud de rechazo sutil, expresado a través de chistes avergonzantes, bromas sexuales, insultos ofensivos, chismes difamatorios $\mathrm{y}$ discriminaciones enmascaradas (Crompton, 2006); ahí radica la importancia de evaluar el rechazo hacia las personas no heterosexuales desde diversos conceptos con mayor o menor contenido de lo sutil.

Campo y Herazo (2008), en una revisión sistemática de los estudios sobre la prevalencia de la actitud de rechazo hacia personas no heterosexuales entre estudiantes de medicina, publicados de 1998 a 2007 en seis bases de datos, se registró una actitud de rechazo entre $10 \mathrm{y}$ $25 \%$ de los estudiantes. Campo, Díaz y Herazo (2008), en una segunda revisión sistemática, hallaron que el promedio de actitud de rechazo hacia personas no heterosexuales entre estudiantes de odontología fue bajo (no rechazo). Campo, Herazo y Cogollo (2010), en un tercer estudio del mismo tipo, desde los resultados de 8 investigaciones, informaron que entre el $7 \mathrm{y}$ $16 \%$ de estudiantes de enfermería rechazaba a las personas no heterosexuales.

EnMéxico, Moraly Martínez-Sulvarán(2012) reportaron una media baja, ubicada dentro de un rango de respuesta en desacuerdo con el rechazo abierto hacia personas no heterosexuales en una muestra de 400 estudiantes de psicología de dos universidades públicas, utilizando una escala de actitud de 10 ítems. En este mismo país, en una muestra de 356 estudiantes de medicina de una universidad privada, Moral y Valle (2012) reportaron una media dentro de un rango de respuesta ambigua entre la aceptación y el rechazo hacia las personas no heterosexuales, utilizando la escala de actitud hacia lesbianas y hombres homosexuales (ATLG, Herek, 1984). La aceptación fue mayor entre los estudiantes de psicología, entre quienes se halla una mayor proporción de mujeres. A sabiendas de que el sexo tiene un efecto significativo en la actitud hacia las personas no heterosexuales, con mayor rechazo en hombres (Herek \& McLemore, 2013), sería importante controlar esta variable a la hora de estudiar las fuentes de variación en estas diferencias.

Para hacer efectivos los derechos de salud que poseen las personas no heterosexuales, se está evaluando la actitud hacia personas no heterosexuales en estudiantes de ciencias de la salud e implementando talleres para facilitar un cambio de actitud hacia la aceptación de la diversidad sexual (Matharu et al., 2012; Selleger et al., 2006). En concordancia con esta línea de investigación, en una muestra de estudiantes de ciencias de la salud, el presente estudio tiene como objetivos: 1) definir dos componentes de rechazo hacia las personas no heterosexuales, uno de rechazo abierto y otro de rechazo 
sutil; 2) calcular las medias de actitud hacia personas no heterosexuales, homonegatividad internalizada, homofobia externalizada y los dos posibles componentes de rechazo abierto y sutil; 3) comparar estas medias entre tres facultades de ciencias de la salud, una pública de medicina, una privada de medicina y otra pública de psicología; y 4) controlar el efecto del sexo en estas comparaciones de medias.

En el presente estudio se contemplan tres conceptos afines de homonegatividad, pero con carga diferencial del rechazo abierto y sutil, de ahí la posibilidad de definir dos componentes de rechazo. La carga del rechazo abierto es mayor en los conceptos de homofobia y actitud de rechazo abierto que en los conceptos de homonegatividad internalizada y actitud de rechazo simbólico; y viceversa con respecto al rechazo sutil. Se hipotetiza que el componente de rechazo abierto se definiría con la escala de homofobia externalizada y las subescalas de actitud de rechazo abierto y rechazo de la manifestación pública de la desviación de la heterosexualidad; por su parte, el componente de rechazo sutil se definiría con las subescalas de actitud de rechazo sutil, rechazo del deseo hacia personas del mismo sexo propio y consideración de las personas homosexuales como incapaces de intimidad.

Se espera que las medias de homofobia externalizada, actitud de rechazo abierto y el componente de rechazo abierto queden en el rango de la aceptación, y que las medias de homonegatividad internalizada, actitud de rechazo simbólico y el componente de rechazo sutil queden en el rango de la ambigüedad en el conjunto de estudiantes, al considerar el cambio experimentado por la actitud hacia las personas no heterosexuales en la cultura occidental contemporánea (Crompton, 2006; Herek, 2004) y estudios previos (Campo, Diaz, \& Herazo, 2008; Campo \& Herazo, 2008; Campo, et al., 2010; Moral \& Martínez-Sulvarán, 2012; Moral \& Valle, 2012).

Se espera un mayor nivel de rechazo hacia las personas no heterosexuales entre los estudiantes de medicina de la universidad privada que entre los estudiantes de psicología de la universidad pública, debido a dos motivos: 1) una mayor proporción de hombres entre los estudiantes de medicina que entre los de psicología, siendo la expectativa de mayor rechazo entre los hombres que entre las mujeres (Herek \& McLemore, 2013), y, 2) posiblemente, por los valores más conservadores (cristiano-demócratas) en las familias de los estudiantes que acuden a las universidades privadas y/o en las instituciones educativas privadas, siendo la expectativa de mayor rechazo entre las personas con valores más conservadores (Pulido et al., 2012). Por la primera razón se espera mayor rechazo en los estudiantes de medicina de la facultad pública, que en los estudiantes de psicología de la facultad pública y, por la segunda razón, se espera menor rechazo entre los estudiantes de medicina de la universidad pública que los de la privada. Además, se prevé que el componente de rechazo sutil sea más diferencial entre las tres facultades que el componente de rechazo abierto debido a que representa una expresión 
del rechazo socialmente más aceptada (Herek, 2004; Herek \& McLemore, 2013).

Se anticipa que todas estas diferencias sean atribuibles a la carrera (psicología versus medicina) y/o universidad (pública versus privada) y no sean totalmente reducibles al sexo.

\section{Método}

\section{Participantes}

Lapoblaciónestuvocompuestaporestudiantes universitarios de medicina y psicología. Se obtuvo una muestra no probabilística de 231 participantes voluntarios: 100 (43\%) fueron encuestados en la Facultad de Medicina de la Universidad Autónoma de Coahuila (UAC), $66(29 \%)$ en la Escuela de Medicina del Tecnológico de Monterrey (ITESM) y 65 (28 \%) en la Facultad de Psicología de la Universidad Autónoma de Nuevo León (UANL).

De los 224 participantes que especificaron su sexo, 121 (54\%) dijeron ser mujeres y 103 (46 $\%)$ hombres, siendo estadísticamente equivalente la frecuencia de ambos sexos (prueba binomial: $p=.26$ ). La media de edad fue de 19.13 años $(D E=1.68)$, con un mínimo de 17 y máximo de 37 . Con respecto a la adscripción religiosa, 182 (79\%) dijeron ser católicos, 10 (4 $\%$ ) cristianos y 39 (17\%) pertenecer a otras religiones o tener creencias religiosas personales. Ninguno se definió como ateo o sin religión. Todos señalaron ser solteros.

\section{Procedimiento}

Los instrumentos de evaluación se administraron de forma auto-aplicada en los salones de clase por los autores del artículo, quienes leían las instrucciones y permanecían en el salón para responder dudas, mantener silencio, recoger los cuestionarios contestados, revisar que los participantes no dejasen preguntas ni escalas sin responder. La aplicación tardaba aproximadamente 15 minutos y se realizó de enero a mayo de 2012.

\section{Instrumentos}

Se evaluó actitud hacia las personas no heterosexuales, homonegatividad internalizada y homofobia externalizada por medio de las siguientes tres escalas:

Escala de actitudes hacia lesbianas y hombres homosexuales (ATLG) de Herek (1984). La escala ATLG se compone de 20 ítems tipo Likert. Cada ítem tiene cinco opciones de respuesta y un rango de 1 a 9 . La suma de los siete ítems de aceptación de los hombres homosexuales y las lesbianas (G1, G5, G7, G9, L2, L4 y L7; puntuados: 1 = "definitivamente de acuerdo", $3=$ "de acuerdo", 5 = "ni de acuerdo ni en desacuerdo", 7 = "en desacuerdo" y $9=$ "completamente en desacuerdo") y los restantes trece ítems invertidos (puntuados: 1 = "completamente en desacuerdo", 3 = "en desacuerdo", 5 = "ni de acuerdo ni en desacuerdo", 7 = "de acuerdo" y $9=$ "definitivamente de acuerdo") proporciona 
una puntación que a mayor valor refleja más rechazo. El rango de la puntuación total es de 20 a 180 . Se ha reportado una estructura de dos factores correlacionados: actitud hacia los hombres homosexuales (ATG) con 10 ítems (de G1 a G10) y actitud hacia las lesbianas (ATL) con diez ítems (de L1 a L10) con ajuste adecuado a los datos y valores de consistencia interna altos para ambos factores (Herek \& McLemore, 2011). La escala fue validada en México por Moral y Valle (2011). En una muestra de 356 estudiantes mexicanos, Moral y Valle (2011) hallaron una consistencia interna alta para los 20 ítems $(\alpha=.94)$ y distribución normal de la puntuación total de la ATLG. Por análisis de componentes principales con rotación Oblimin, fijando el número de factores por el criterio de Kaiser, se obtuvo un factor de rechazo hacia las lesbianas (ATL) con diez ítems (de L1 a L10; por ejemplo, "la homosexualidad femenina es mala para nuestra sociedad porque rompe la división natural entre los sexos") con consistencia interna alta $(\alpha=.91)$, otro factor de rechazo abierto hacia los hombres homosexuales (ATG-A) con cinco ítems (G2, G3, G4, G6 y G10; por ejemplo, “a los hombres homosexuales no debería permitírseles enseñar en los colegios") con consistencia interna alta $(\alpha=.85)$ y otro factor de rechazo sutil hacia los hombres homosexuales (ATG-S) con cinco ítems (G1, G5, G7, G8 y G9; por ejemplo, "la idea del matrimonio homosexual me parece ridícula") con consistencia interna alta $(\alpha=.78)$. Esta estructura de tres factores correlacionados tuvo un ajuste adecuado a los datos $(\chi 2 / g l=$ 2.11, FD =0.99, $P N C P=0.52, G F I=.90, A G F I$ $=.88$ y $R M S E A=.06)$ por mínimos cuadrados generalizados (Moral \& Valle, 2011). En el presente estudio se usaron las tres subescalas del estudio de validación en México (ATL, ATG-A y ATG-S), aparte de la puntuación total (ATLG).

La escala de Homonegatividad Internalizada (HNI-16) de Moral y Valle (2013a) está integrada por 16 ítems tipo Likert con cinco opciones de respuesta y un rango de 1 a 9 ( $1=$ "totalmente en desacuerdo", 3 = "en desacuerdo", 5 = "ni de acuerdo ni en desacuerdo", 7 = "de acuerdo" y 9 = "totalmente de acuerdo"). La puntuación total se obtiene por suma simple de los doce ítems tras invertir las puntuaciones de los cinco ítems redactados en sentido de aceptación o inversos (ítems 1, 2, 3, 4 y 11). Su consistencia interna fue alta $(\alpha=.88)$ y quedó integrada por tres factores: rechazo de la manifestación pública de la homosexualidad (EXT) con seis ítems (por ejemplo, "las situaciones sociales con hombres homosexuales me hace sentir incómodo") con consistencia interna alta $(\alpha=.81)$; rechazo de sentimientos, deseos e identidad homosexuales propios (INT) también con seis (por ejemplo, "yo me sentiría contrariado, fuera de mí mismo, debido a la presencia de sentimientos homosexuales en mí") con consistencia interna alta $(\alpha=.81)$, e incapacidad de las personas homosexuales para la intimidad (PROMI) con cuatro ítems (por ejemplo, "los hombres homosexuales tienden a mostrar su sexualidad inapropiadamente") con consistencia interna 
adecuada $(\alpha=.69)$. Un modelo de tres factores jerarquizados a uno general mostró un ajuste a los datos que varió de bueno $(\chi 2 / g l=1.66$, $P N C P=0.29$ y $R M S E A=.05)$ a adecuado $(F D$ $=0.73, G F I=.91$ y $A G F I=.88)$ por mínimos cuadrados generalizados. La distribución de su puntuación total se ajustó a una curva normal, al igual que la de sus factores EXT e INT (Moral \& Valle, 2013a). En el presente estudio se usaron las tres subescalas (INT, EXT y PROMI), aparte de la puntuación total.

La Escala de Homofobia (HF-8) de Klamen, Grossman y Kopacz (1999) con la adaptación mexicana de Moral y Valle (2013b), está integrada por ocho ítems tipo Likert (por ejemplo, "la homosexualidad es inmoral") con cuatro opciones de respuestas y un rango de 1 a 7 (1 = "completamente en desacuerdo", 3 = "en desacuerdo", 5 = "de acuerdo" y 7 = "definitivamente de acuerdo"). La consistencia interna de estos ocho ítems fue alta $(\alpha=.84)$ y el modelo unifactorial mostró índices de ajuste a los datos de bueno $(G F I=.95, A G F I=.91, P N C P=0.11$ y $R M S E A=.07$, siendo $p=.08$ para la hipótesis nula de $R M S E A \leq .05)$ a adecuados $(\chi 2 / g l=2.28$ y $F D=0.19)$ por mínimos cuadrados generalizados. La distribución de la puntuación total de la escala HF-8, con un rango de 10 a 56 y media de $23.02(D E=8.86)$, se ajustó a una curva normal $\left(Z_{K-S}=1.24, p=.09\right)$. La puntuación total se obtuvo por la suma simple de los ocho ítems tras invertir la puntuación de dos de ellos (ítems 1 y 4). Mayor puntuación en la escala HF-8 refleja mayor homofobia externalizada o condena de la desviación de la heterosexualidad (Moral \& Valle, 2013b). En el presente estudio se usó la puntuación total al ser la escala HF-8 unidimensional.

\section{Procedimiento}

Se realizó un estudio descriptivo-correlacional con un diseño ex post-facto transversal. Se solicitó el consentimiento informado para la participación en el estudio de forma verbal, garantizando el anonimato y confidencialidad de la información de acuerdo con las normas éticas de investigación de la Sociedad Mexicana de Psicología (2007).

\section{Análisis de datos}

El análisis de componentes principales se aplicó a la matriz de correlaciones entre las tres subescalas de HNI-16, las tres subescalas de ATLG y la escala HF-8. El número de componentes se determinó por la expectativa: dos. La matriz de componentes se rotó por el método Oblimin directo y las puntuaciones en los componentes se calcularon por el método de regresión. Se consideraron las saturaciones en la matriz de configuraciones $\geq .50$ como altas.

Las comparaciones de medias de las tres puntuaciones totales, seis subescalas y dos componentes entre las tres facultades se hicieron por medio de un análisis de varianza (ANOVA) para muestras independientes. El tamaño del efecto se calculó por el estadístico $f^{2}$ de Cohen. El tamaño del efecto se consideró pequeño si .02 $\leq$ $f^{2}<.15$, mediano si $15 \leq f^{2}<.35$ y grande si $f^{2}$ $\geq .35$ (Ellis, 2010). La equivalencia de varianza entre las tres muestras se contrastó por la prueba 
de Levene. Se hicieron las comparaciones de medias por pares de muestras (comparaciones post doc) por medio de la prueba de la diferencia mínima significativa (DSM) de Fisher, al cumplirse el supuesto de varianzas equivalentes entre los tres grupos. Finalmente, se parcializó el efecto del sexo en las diferencias significativas con el fin de determinar en qué grado la diferencia era efecto del sexo. Esta parcialización se realizó por análisis de covarianza (ANCOVA). El nivel de significación se estipuló en .05. Los cálculos estadísticos fueron realizados con el programa SPSS versión 16.

\section{Resultados}

\section{Definición de los componentes de rechazo abierto y rechazo sutil}

Desde la distinción entre un rechazo abierto/ externo y un rechazo sutil/interno, se esperarían dos compontes. Al extraer dos componentes de la matriz de correlaciones de los siete factores de las tres escalas usadas en el presente estudio (ATG-A, ATG-S, ATL, HF-8, EXT, INT y PROMI), se explicó $74.9 \%$ de la varianza total. Tras la rotación oblicua (por el método Oblimin directo), se configuró un primer componente con tres factores de ATGL y HF-8 y un segundo componente con los tres factores de HNI-16, lo cual no concordó con la expectativa.

Al eliminar PROMI y EXT, sí se obtuvo el resultado esperado. Los dos componentes explicaron $84.9 \%$ de la varianza total. Tras la rotación oblicua, el primer componente quedó configurado por ATG-A, HF-8 y ATL, además, su contenido correspondió a rechazo abierto/externo. El segundo componente quedó configurado por ATG-S e INT y su contenido correspondió a rechazo sutil/interno (Véase tabla 1). La magnitud de la asociación entre los componentes fue alta $(r=.59, p<.01)$.

Tabla 1

Matriz de configuraciones con cargas factoriales mayores o iguales que .50

\begin{tabular}{llll}
\hline \multirow{2}{*}{ Subescalas } & \multicolumn{2}{l}{ Componentes } & Comunalidad \\
\cline { 2 - 4 } & 1 & 2 & \\
\hline ATG-A & .971 & & .851 \\
HF-8 & .892 & & .809 \\
ATL & .841 & .996 & .833 \\
INT & & .580 & .936 \\
ATG-S & & .817 \\
\hline
\end{tabular}

Método de extracción: Análisis de componentes principales. Método de rotación: Normalización Oblimin con Kaiser. La rotación convergió en 5 iteraciones. Subescalas: ATG-A = Rechazo abierto de los hombres homosexuales, HF-8 = Suma total escala HF con 8 ítems, ATL = Rechazo hacia las lesbianas, INT = Rechazo de los sentimientos, deseos e identidad homosexuales propios, y ATG-S = Rechazo sutil de los hombres homosexuales. 


\section{Medias de rechazo hacia personas no hetero- sexuales en la muestra total de estudiantes}

Para interpretar las medias de cada puntuación total de escala o de cada subescala $(M)$ se dividió entre el número de ítems de la respectiva escala o subescala $(N I)$. De este modo, se redujeron los rangos de las escalas y subescalas al rango de valores discretos de los ítems; 1, 3, 5, 7 y 9 ó 1, 3, 5 y 7. Para las medias de la escalas ATLG y HNI-16 y sus subescalas ATG-A, ATG-S, ATL, EXT, INT y PROMI, se consideraron cinco intervalos de amplitud constante en correspondencia con los cinco valores discretos de respuesta a los ítems (amplitud del intervalo $=[$ valor máximo - valor mínimo $] /$ número valores de respuesta al ítem $=[9-1] / 5=1.6)$ : valores continuos de 1 a 2.6 corresponden al valor discreto $1=$ "definitivamente en desacuerdo", de 2.61 a 4.2 corresponden al valor discreto 3 = "en desacuerdo", de 4.21 a 5.8 corresponden al valor discreto 5 = "ambigüedad entre el acuerdo y el desacuerdo", de 5.81 a 7.4 corresponden al valor discreto $7=$ "de acuerdo" y de 7.41 a 9 corresponden al valor discreto $9=$ “completamente de acuerdo". Para la puntuación total de la escala HF-8, se consideraron cuatro intervalos de amplitud constante en correspondencia con los cuatro valores discretos de respuesta a los ítems (amplitud del intervalo $=[$ valor máximo - valor mínimo $] /$ número valores de respuesta al ítem $=[7-1] / 4=1.5)$ : valores continuos de 1 a 2.5 corresponden al valor discreto $1=$ "definitivamente en desacuerdo", de 2.51 a 4 corresponden al valor discreto 3 = "en desacuerdo", de 4.01 a 5.5 corresponden al valor discreto 5 = "de acuerdo", de 5.51 a 7 corresponden al valor discreto $7=$ "completamente de acuerdo".

Las medias de las escalas HF-8 $(M / N I=2.88)$ y $\operatorname{ATLG}(M / N I=3.54)$ y las subescalas ATG-A $(M / N I=2.97), \operatorname{ATL}(M / N I=3.11)$ y $\operatorname{EXT}(M / N I$ $=3.92$ ), correspondieron a valores discretos de 3 = "en desacuerdo" con el rechazo. La media de la escala HNI-16 $(M / N I=4.60)$ y las subescalas PROMI $(M / N I=4.74)$, ATG-S $(M / N I=$ 4.95) e INT $(M / N I=5.19)$, correspondieron a valores discretos de $5=$ "ambigüedad entre la aceptación y el rechazo".

Al calcular la media de las medias de las subescalas ATG-S y INT (componente de rechazo sutil/interno), se obtuvo un valor de 5.07, que se ubicó en el intervalo [4.21, 5.8], correspondiente a una respuesta de ambigüedad entre la aceptación y el rechazo (valor discreto 5). Al calcular la media de las medias de las subescalas ATG-A, ATL y la escala HF-8 (componente de rechazo abierto/externo), se obtuvo un valor de 2.99, que se ubicó en el intervalo [2.61, 4.2], correspondiente a una respuesta de desacuerdo con el rechazo (valor discreto 3 ).

Al calcular la media de las medias de las escalas ATLG, HNI-16 y HF-8 y sus seis factores (ATG-A, ATG-S, ATL, EXT, INT y PROMI) se obtuvo un valor de 3.99, que se ubicó en el intervalo [2.61, 4.2], correspondiente a una respuesta de desacuerdo con el rechazo (valor discreto 3 ). 


\section{Comparación de medias entre las tres facul-} tades

Al comparar las medias entre las tres facultades por ANOVA, se obtuvo una equivalencia estadística entre ellas, salvo en el factor de rechazo sutil hacia los hombres homosexuales (ATG-S) y rechazo de la manifestación pública de la homosexualidad (EXT). Los tamaños del efecto de la facultad sobre ATG-S y EXT fueron pequeños (véase tabla 2).

Tabla 2

Descriptivos, contraste de la igualdad de varianza y medias entre las tres facultades

\begin{tabular}{|c|c|c|c|c|c|c|c|c|c|c|c|}
\hline \multirow{2}{*}{ Escala } & \multirow{2}{*}{ Facultad } & \multirow{2}{*}{$N$} & \multirow{2}{*}{$M$} & \multirow{2}{*}{$D E$} & \multicolumn{2}{|c|}{ Levene* } & \multicolumn{2}{|c|}{ ANOVA* } & \multicolumn{3}{|c|}{ Tamaño del efecto } \\
\hline & & & & & $F$ & $p$ & $F$ & $p$ & $\eta$ & $\eta^{2}$ & $f^{2}$ \\
\hline ATLG & $\begin{array}{l}\text { Med-priv } \\
\text { Med-publ } \\
\text { Psico-publ }\end{array}$ & $\begin{array}{l}66 \\
100 \\
65\end{array}$ & $\begin{array}{l}70.91 \\
72.72 \\
67.57\end{array}$ & $\begin{array}{l}30.52 \\
30.40 \\
23.85\end{array}$ & 2.65 & .073 & 0.63 & .532 & .074 & .006 & .006 \\
\hline ATG-A & $\begin{array}{l}\text { Med-priv } \\
\text { Med-publ } \\
\text { Psico-publ }\end{array}$ & $\begin{array}{l}66 \\
100 \\
65\end{array}$ & $\begin{array}{l}14.61 \\
15.20 \\
14.57\end{array}$ & $\begin{array}{l}7.65 \\
7.58 \\
7.06\end{array}$ & 0.54 & .586 & 0.19 & .826 & .041 & .002 & .002 \\
\hline ATG-S & $\begin{array}{l}\text { Med-priv } \\
\text { Med-publ } \\
\text { Psico-publ }\end{array}$ & $\begin{array}{l}66 \\
100 \\
65\end{array}$ & $\begin{array}{l}25.24 \\
26.24 \\
22.02\end{array}$ & $\begin{array}{l}9.64 \\
9.69 \\
8.39\end{array}$ & 2.15 & .119 & 4.16 & .017 & .188 & .035 & .034 \\
\hline ATL & $\begin{array}{l}\text { Med-priv } \\
\text { Med-publ } \\
\text { Psico-publ }\end{array}$ & $\begin{array}{l}66 \\
100 \\
65\end{array}$ & $\begin{array}{l}31.06 \\
31.28 \\
30.98\end{array}$ & $\begin{array}{l}16.39 \\
15.22 \\
12.49\end{array}$ & 2.31 & .101 & 0.01 & .991 & .009 & $<.001$ & $<.001$ \\
\hline $\mathrm{HNI}-16$ & $\begin{array}{l}\text { Med-priv } \\
\text { Med-publ } \\
\text { Psico-publ } \\
\text { Total }\end{array}$ & $\begin{array}{l}66 \\
100 \\
65 \\
231\end{array}$ & $\begin{array}{l}74.55 \\
75.68 \\
69.35 \\
73.58\end{array}$ & $\begin{array}{l}21.97 \\
22.75 \\
19.63 \\
21.76\end{array}$ & 1.03 & .358 & 1.77 & .173 & .124 & .015 & .015 \\
\hline INT & $\begin{array}{l}\text { Med-priv } \\
\text { Med-Publ } \\
\text { Psico-publ }\end{array}$ & $\begin{array}{l}66 \\
100 \\
65\end{array}$ & $\begin{array}{l}32.45 \\
31.78 \\
28.74\end{array}$ & $\begin{array}{l}10.70 \\
10.54 \\
9.18\end{array}$ & 0.90 & .407 & 2.53 & .082 & .147 & .022 & .022 \\
\hline EXT & $\begin{array}{l}\text { Med-priv } \\
\text { Med-publ } \\
\text { Psico-publ }\end{array}$ & $\begin{array}{l}66 \\
100 \\
65\end{array}$ & $\begin{array}{l}23.55 \\
25.04 \\
21.17\end{array}$ & $\begin{array}{l}8.970 \\
10.18 \\
9.84\end{array}$ & 1.03 & .358 & 3.10 & .047 & .163 & .027 & .028 \\
\hline PROMI & $\begin{array}{l}\text { Med-priv } \\
\text { Med-publ } \\
\text { Psico-publ }\end{array}$ & $\begin{array}{l}231 \\
100 \\
65\end{array}$ & $\begin{array}{l}23.52 \\
18.86 \\
19.45\end{array}$ & $\begin{array}{l}9.84 \\
6.06 \\
5.73\end{array}$ & 1.35 & .262 & 0.37 & .691 & .057 & .003 & .003 \\
\hline HF-8 & $\begin{array}{l}\text { Med-priv } \\
\text { Med-publ } \\
\text { Psico-publ }\end{array}$ & $\begin{array}{l}66 \\
100 \\
65\end{array}$ & $\begin{array}{l}22.18 \\
23.58 \\
23.02\end{array}$ & $\begin{array}{l}9.11 \\
9.22 \\
8.06\end{array}$ & 1.23 & .294 & 0.49 & .611 & .066 & .004 & .004 \\
\hline $\begin{array}{l}\text { Rechazo } \\
\text { Abierto }\end{array}$ & $\begin{array}{l}\text { Med-priv } \\
\text { Med-publ } \\
\text { Psico-publ }\end{array}$ & $\begin{array}{l}66 \\
100 \\
65\end{array}$ & $\begin{array}{l}-0.05 \\
0.06 \\
-0.04\end{array}$ & $\begin{array}{l}1.05 \\
1.05 \\
0.86\end{array}$ & 2.37 & .095 & 0.29 & .748 & .050 & .003 & .003 \\
\hline $\begin{array}{l}\text { Rechazo } \\
\text { Sutil }\end{array}$ & $\begin{array}{l}\text { Med-priv } \\
\text { Med-publ } \\
\text { Psico-publ }\end{array}$ & $\begin{array}{l}66 \\
100 \\
65\end{array}$ & $\begin{array}{l}0.13 \\
0.10 \\
-0.29\end{array}$ & $\begin{array}{l}1.06 \\
1.01 \\
0.86\end{array}$ & 1.42 & .245 & 3.86 & .023 & .181 & .033 & .034 \\
\hline
\end{tabular}

* Grados de libertad: $g l_{1}=2, g l_{2}=228$. Escala: $A T L G=$ Suma total de la escala ATLG, ATG-A = Rechazo abierto de los hombres homosexuales, ATG- $\mathrm{S}=$ Rechazo sutil de los hombres homosexuales, ATL = Rechazo hacia las lesbianas, HNI = Suma total de la escala de HNI con 16 ítems, INT = Rechazo de los sentimientos, deseos e identidad homosexuales propios, EXT = Rechazo de la manifestación pública de la homosexualidad, PROMI = Incapacidad para relaciones estables, HF-8 = Suma total escala HF con 8 ítems. Rechazo abierto/externo = Puntuaciones estandarizadas del componente configurado por ATG-A, ATL y HF-8. Rechazo sutil/interno = Puntuaciones estandarizadas del componente configurado por ATG-S e INT. Facultades: Med-priv = facultad medicina privada en Monterrey, Med-publ = facultad de medicina pública en Saltillo y Psico-publ = facultad de psicología pública en Monterrey. 
Tras realizar las comparaciones por pares (post doc), con respecto a ATG-S se obtuvo que las medias de los estudiantes de las dos facultades de medicina fueron estadísticamente equivalentes entre sí y estadísticamente mayores que las medias de los estudiantes de la facultad de psicología. Con respecto a EXT se obtuvo que los estudiantes de medicina de la universidad pública tuvieron una media significativamente más alta que los estudiantes de psicología, no se registró diferencia significativa en las otras dos comparaciones de medias (Véase tabla 3).

Tabla 3

Comparaciones de medias por pares por la prueba de la diferencia mínima significativa de Fisher

\begin{tabular}{|c|c|c|c|c|c|c|c|}
\hline \multirow{2}{*}{$\begin{array}{l}\text { Variable } \\
\text { Comparada }\end{array}$} & \multirow{2}{*}{$(\mathrm{I})-(\mathrm{J})$} & & \multirow{2}{*}{ Media (I-J) } & \multirow{2}{*}{$E E$} & \multirow{2}{*}{$p$} & \multicolumn{2}{|l|}{$95 \%$ IC } \\
\hline & & & & & & $L I$ & $L S$ \\
\hline ATLG & $\begin{array}{l}\text { Med-priv } \\
\text { Med-publ }\end{array}$ & $\begin{array}{l}\text { Med-publ } \\
\text { Psico-publ } \\
\text { Psico-publ }\end{array}$ & $\begin{array}{l}-1.81 \\
3.34 \\
5.15\end{array}$ & $\begin{array}{l}4.56 \\
5.02 \\
4.58\end{array}$ & $\begin{array}{l}.692 \\
.507 \\
.262\end{array}$ & $\begin{array}{l}-10.80 \\
-6.56 \\
-3.88\end{array}$ & $\begin{array}{l}7.17 \\
13.24 \\
14.18\end{array}$ \\
\hline ATG-A & $\begin{array}{l}\text { Med-priv } \\
\text { Med-publ }\end{array}$ & $\begin{array}{l}\text { Med-publ } \\
\text { Psico-publ } \\
\text { Psico-publ }\end{array}$ & $\begin{array}{l}-0.59 \\
0.04 \\
0.63\end{array}$ & $\begin{array}{l}1.18 \\
1.30 \\
1.19\end{array}$ & $\begin{array}{l}.616 \\
.977 \\
.596\end{array}$ & $\begin{array}{l}-2.92 \\
-2.53 \\
-1.71\end{array}$ & $\begin{array}{l}1.74 \\
2.60 \\
2.97\end{array}$ \\
\hline ATG-S & $\begin{array}{l}\text { Med-priv } \\
\text { Med-publ }\end{array}$ & $\begin{array}{l}\text { Med-publ } \\
\text { Psico-publ } \\
\text { Psico-publ }\end{array}$ & $\begin{array}{l}-0.99 \\
\mathbf{3 . 2 3}^{*} \\
\mathbf{4 . 2 3}^{*}\end{array}$ & $\begin{array}{l}1.48 \\
1.63 \\
1.49\end{array}$ & $\begin{array}{l}.501 \\
.049 \\
.005\end{array}$ & $\begin{array}{l}-3.91 \\
0.01 \\
1.30\end{array}$ & $\begin{array}{l}1.92 \\
6.44 \\
7.15\end{array}$ \\
\hline ATL & $\begin{array}{l}\text { Med-priv } \\
\text { Med-publ }\end{array}$ & $\begin{array}{l}\text { Med-publ } \\
\text { Psico-publ } \\
\text { Psico-publ }\end{array}$ & $\begin{array}{l}-0.22 \\
0.08 \\
0.30\end{array}$ & $\begin{array}{l}2.36 \\
2.60 \\
2.37\end{array}$ & $\begin{array}{l}.926 \\
.977 \\
.901\end{array}$ & $\begin{array}{l}-4.86 \\
-5.04 \\
-4.37\end{array}$ & $\begin{array}{l}4.43 \\
5.19 \\
4.96\end{array}$ \\
\hline $\mathrm{HNI}-16$ & $\begin{array}{l}\text { Med-priv } \\
\text { Med-publ }\end{array}$ & $\begin{array}{l}\text { Med-publ } \\
\text { Psico-publ } \\
\text { Psico-publ }\end{array}$ & $\begin{array}{l}-1.14 \\
5.19 \\
6.33\end{array}$ & $\begin{array}{l}3.44 \\
3.79 \\
3.46\end{array}$ & $\begin{array}{l}.742 \\
.172 \\
.068\end{array}$ & $\begin{array}{l}-7.91 \\
-2.28 \\
-0.48\end{array}$ & $\begin{array}{l}5.64 \\
12.66 \\
13.14\end{array}$ \\
\hline INT & $\begin{array}{l}\text { Med-priv } \\
\text { Med-publ }\end{array}$ & $\begin{array}{l}\text { Med-publ } \\
\text { Psico-publ } \\
\text { Psico-publ }\end{array}$ & $\begin{array}{l}0.68 \\
3.72^{*} \\
3.04\end{array}$ & $\begin{array}{l}1.62 \\
1.79 \\
1.63\end{array}$ & $\begin{array}{l}.678 \\
.039 \\
.063\end{array}$ & $\begin{array}{l}-2.52 \\
0.20 \\
-0.17\end{array}$ & $\begin{array}{l}3.87 \\
7.24 \\
6.25\end{array}$ \\
\hline EXT & $\begin{array}{l}\text { Med-priv } \\
\text { Med-publ }\end{array}$ & $\begin{array}{l}\text { Med-publ } \\
\text { Psico-publ } \\
\text { Psico-publ }\end{array}$ & $\begin{array}{l}-1.49 \\
2.38 \\
3.87^{*}\end{array}$ & $\begin{array}{l}1.55 \\
1.70 \\
\mathbf{1 . 5 5}\end{array}$ & $\begin{array}{l}.335 \\
.165 \\
.013\end{array}$ & $\begin{array}{l}-4.54 \\
-0.98 \\
\mathbf{0 . 8 1}\end{array}$ & $\begin{array}{l}1.55 \\
5.73 \\
6.93\end{array}$ \\
\hline PROMI & $\begin{array}{l}\text { Med-priv } \\
\text { Med-publ }\end{array}$ & $\begin{array}{l}\text { Med-publ } \\
\text { Psico-publ } \\
\text { Psico-publ }\end{array}$ & $\begin{array}{l}-0.32 \\
-0.90 \\
-0.59\end{array}$ & $\begin{array}{l}0.97 \\
1.07 \\
0.97\end{array}$ & $\begin{array}{l}.746 \\
.399 \\
.547\end{array}$ & $\begin{array}{l}-2.22 \\
-3.00 \\
-2.50\end{array}$ & $\begin{array}{l}1.59 \\
1.20 \\
1.33\end{array}$ \\
\hline HF-8 & $\begin{array}{l}\text { Med-priv } \\
\text { Med-publ }\end{array}$ & $\begin{array}{l}\text { Med-publ } \\
\text { Psico-publ } \\
\text { Psico-publ }\end{array}$ & $\begin{array}{l}-1.40 \\
-0.83 \\
0.57\end{array}$ & $\begin{array}{l}1.41 \\
1.55 \\
1.41\end{array}$ & $\begin{array}{l}.322 \\
.591 \\
.690\end{array}$ & $\begin{array}{l}-4.17 \\
-3.89 \\
-2.22\end{array}$ & $\begin{array}{l}1.38 \\
2.22 \\
3.35\end{array}$ \\
\hline $\begin{array}{l}\text { Rechazo } \\
\text { abierto }\end{array}$ & $\begin{array}{l}\text { Med-priv } \\
\text { Med-publ }\end{array}$ & $\begin{array}{l}\text { Med-publ } \\
\text { Psico-publ } \\
\text { Psico-publ }\end{array}$ & $\begin{array}{l}-0.11 \\
-0.01 \\
0.09\end{array}$ & $\begin{array}{l}0.16 \\
0.18 \\
0.16\end{array}$ & $\begin{array}{l}.498 \\
.934 \\
.559\end{array}$ & $\begin{array}{l}-0.42 \\
-0.36 \\
-0.22\end{array}$ & $\begin{array}{l}0.21 \\
0.33 \\
0.41\end{array}$ \\
\hline $\begin{array}{l}\text { Rechazo } \\
\text { sutil }\end{array}$ & $\begin{array}{l}\text { Med-priv } \\
\text { Med-publ }\end{array}$ & $\begin{array}{l}\text { Med-publ } \\
\text { Psico-publ } \\
\text { Psico-publ }\end{array}$ & $\begin{array}{l}0.02 \\
0.42^{*} \\
\mathbf{0 . 3 9}^{*}\end{array}$ & $\begin{array}{l}0.16 \\
\mathbf{0 . 1 7} \\
\mathbf{0 . 1 6}\end{array}$ & $\begin{array}{l}.882 \\
.017 \\
.014\end{array}$ & $\begin{array}{l}-0.28 \\
\mathbf{0 . 0 7} \\
\mathbf{0 . 0 8}\end{array}$ & $\begin{array}{l}0.33 \\
0.76 \\
0.70\end{array}$ \\
\hline
\end{tabular}

Escala: $A T L G=$ Suma total de la escala ATLG, ATG-A = Rechazo abierto de los hombres homosexuales, ATG- $S=$ Rechazo sutil de los hombres homosexuales, $\mathrm{ATL}=$ Rechazo hacia las lesbianas, $\mathrm{HNI}=$ Suma total de la escala de $\mathrm{HNI}$ con 16 ítems, INT = Rechazo de los sentimientos, deseos e identidad homosexuales propios, EXT = Rechazo de la manifestación pública de la homosexualidad, $\mathrm{PROMI}=$ Incapacidad para relaciones estables, HF-8 = Suma total escala HF con 8 ítems. Rechazo abierto/externo = Puntuaciones estandarizadas del componente configurado por ATG-A, ATL y HF-8. Rechazo sutil/interno = Puntuaciones estandarizadas del componente configurado por ATG-S e INT. Facultades: Med-priv = facultad medicina privada en Monterrey, Med-publ = facultad de medicina pública en Saltillo y Psico-publ = facultad de psicología pública en Monterrey. 
Aunque las diferencias no fueron estadísticamente significativas en las otras subescalas de HNI-16 y ATLG, conforme a la expectativa, las medias más altas se observaron en los estudiantes de las dos facultades de medicina y las medias más bajas en los estudiantes de la facultad de psicología (Véase tabla 2). Incluso, la media de los estudiantes de la facultad de medicina privada en el factor de aceptación de los sentimientos, deseos e identidad homosexuales propios (INT) fue significativamente mayor que la media de los estudiantes de la facultad de psicología pública (Véase tabla 3).

Se obtuvieron las puntuaciones en los dos componentes por el método de regresión. Al comparar las medias de estos dos componentes entre las tres facultades, se obtuvo diferencia significativa en el componente de rechazo sutil/ interno. El tamaño del efecto de la facultad sobre el rechazo sutil fue pequeño (Véase tabla 2). En las comparaciones por pares (post doc), las medias de los estudiantes de las dos facultades de medicina fueron estadísticamente equivalentes entre sí y estadísticamente mayores que la media de los estudiantes de la facultad de psicología (Véase tabla 3).

\section{Parcialización del efecto del sexo}

Tras parcializar el efecto del sexo por ANCOVA, la diferencia de medias en ATG-S entre las tres facultades siguió siendo significativa ( $F$ $[2,220]=4.26, p=.015)$, pero la diferencia de medias en EXT entre las tres facultades perdió significación $(F[2,220]=2.67, p=.072)$. La diferencia en el componente de rechazo sutil/interno también continuó siendo estadísticamente significativa $(F[2,220]=5.29, p=.006)$ como en ATG-S. Por otra parte, se observó que la diferencia de medias en INT entre las tres facultades pasó a ser estadísticamente significativa $(F[2$, $220]=3.76, p=.025)$, cuando en un inicio no lo era $(p=.082)$.

\section{Discusión}

En relación con el primer objetivo de definir dos componentes de rechazo, sí se logró obtener un componente de rechazo abierto/externo y otro de rechazo sutil/interno conforme con la expectativa (Herek, 2004; Herek \& McLemore, 2013); el primero con tres indicadores y el segundo con dos. No obstante, se requirió excluir dos subescalas de la HNI-16 para su logro, ya que no se definía un componente asociado con las subescalas de la ATLG y escala HF-8 y otro componente asociado con las subescalas de la HNI-16. Por lo tanto, los datos prestan sustento empírico a la diferencia cualitativa de dos tipos de rechazo, un rechazo manifiesto o abierto y dirigido hacia el exterior frente a otro rechazo encubierto o sutil en su expresión externa, que aparte de dirigirse hacia el exterior también se dirige hacia el interior. No son dos niveles de intensidad (alta y baja) dentro de un continuo de rechazo, sino dos dimensiones diferenciadas, aunque finalmente con una magnitud de asociación alta, al compartir más o menos un tercio de la varianza. 
En relación con el segundo objetivo de describir los niveles de rechazo hacia las personas no heterosexuales, como se esperaba en estos estudiantes de ciencias de la salud, el posicionamiento en los aspectos manifiestos y públicos (HF-8, EXT, ATG-A, ATLG, ATL, PROMI y componente de rechazo abierto/externo) es de aceptación, puesto que muestran desacuerdo con el rechazo; en tanto, el posicionamiento en los aspectos sutiles o internos (ATG-S, INT, HNI-16 y componente de rechazo sutil/interno) es de ambigüedad entre la aceptación y el rechazo. En concordancia con el cambio de actitud en la cultura occidental a partir de la década de 1970 (Crompton, 2006; Herek, 2004) y, especialmente, en los ámbitos académicos (Morrison et al., 2009), la desviación de la heterosexualidad no es condenada, atacada o perseguida abiertamente; empero, persiste un rechazo en aspectos sutiles y hacia el propio deseo interno. Autores como Burn, Kadlec y Rexer (2005) y Szymanski (2008) sostienen que esta persistencia del rechazo está determinada por la ideología heterosexista dominante. Esta ideología proclama que la heterosexualidad es la única orientación sexual necesaria para el correcto funcionamiento social, contempla a las personas no heterosexuales como inferiores a las heterosexuales y ubica cualquier expresión desviada de la heterosexualidad en una posición marginal (Majied, 2008); de este modo, el rechazo sutil margina algunos derechos (educar niños, matrimonio, herencias, adopción) y quita rango social a las personas no heterosexuales, así, la desviación de la heterosexualidad es un medio de difamación y un contenido fácil de chistes y bromas, sobre todo, en el hombre. Debido a que no se evaluó heterosexismo en el presente estudio no se pudo contrastar su dominancia entre estos estudiantes y su asociación con rechazo sutil.

La diferencia entre los dos estudios realizados en México y citados en la introducción, uno en estudiantes de psicología (Moral \& MartínezSulvarán, 2012) y otro en estudiantes de medicina (Moral \& Valle, 2012), procede de cuatro fuentes: 1) la escala aplicada a los estudiantes de psicología tenía un mayor contenido del rechazo manifiesto (que suscita menos rechazo) que la aplicada a los estudiantes de medicina, 2) los estudiantes de la licenciatura de psicología estaban expuestos a la influencia de programas de sexualidad (que facilitan la aceptación) cuando no era el caso de los estudiantes de medicina, 3) los estudiantes de psicología procedían de universidades públicas y los estudiantes de medicina procedían de una universidad privada, la cual pudiera tener valores más conservadores (que incita a más rechazo) y 4) la proporción de sexo femenino (que facilita la aceptación) era mayor entre estudiantes de psicología.

El presente estudio comparte características con ambos estudios mexicanos. Se aplicaron tres escalas, dos de ellas con mayor contenido del rechazo abierto (HF-8 y ATGL) y una con mayor contenido del rechazo sutil (HNI-16); la muestra quedó integrada por estudiantes de medicina (72\%) y psicología (28\%), inscritos 
en instituciones educativas públicas $(71 \%)$ y privadas (29\%); además, los estudiantes de licenciatura de psicología sí estaban expuestos a la presencia de programas de sexualidad, pero no los de medicina. De ahí que los promedios de rechazo de la presente muestra se ubiquen entre los estudios de Moral y Martínez-Sulvarán (2012) y Moral y Valle (2012), siendo el promedio total de aceptación, como también Campo et al. (2008) reportaron en su estudio de revisión en estudiantes de odontología.

En relación con el tercer objetivo de comparación del rechazo entre tres facultades de ciencias de la salud, se observaron diferencias significativas, con tamaños del efecto pequeños, en los factores de rechazo sutil hacia los hombres homosexuales (ATG-S), rechazo de la manifestación pública de la homosexualidad (EXT) y componente de rechazo sutil/interno. Además, el factor de fantasías, deseos e identidad homosexuales propios (INT) fue diferencial una vez parcializado el efecto del sexo, e incluso este factor fue diferencial en la comparación entre estudiantes de medicina de la universidad privada y estudiantes de psicología de la universidad pública sin parcializar el efecto del sexo. Se confirma la expectativa de mayor rechazo entre los estudiantes de medicina que de psicología en estas diferencias significativas y la tendencia de los promedios en las diferencias no significativas también resultó conforme con esta hipótesis. Dichas diferencias se presentan tanto frente a los estudiantes de medicina de la universidad pública como frente a los estudiantes de medicina de la universidad privada, por lo tanto, no son atribuibles al hecho de que la facultad de psicología sea pública.

Se había hipotetizado que los valores serían más conservadores (cristiano-demócratas) en las familias de los estudiantes que acuden a las universidades privadas y/o en las instituciones educativas privadas, siendo la expectativa de mayor rechazo entre las personas con valores más conservadores (Pulido et al., 2012). Consecuentemente, se esperaba mayor rechazo entre los estudiantes de medicina de la universidad privada que los de la pública. Sin embargo, esto no se cumplió. Sus medias fueron estadísticamente equivalentes, quizá debido a dos motivos. En primer lugar, la variabilidad en la ciudad. Monterrey es una ciudad más abierta y cosmopolita que la ciudad de Saltillo. La facultad de medicina privada se ubica en Monterrey y la pública en Saltillo. Varios estudios indican que el lugar de crianza y/o el hecho de residir en una gran ciudad frente a una ciudad pequeña tienen un efecto significativo en la actitud hacia las personas no heterosexuales; el rechazo es mayor en pueblos y ciudades pequeñas (Hsueh-Hao, 2009; Wienke \& Gretchen, 2013). El posible efecto liberal del tamaño y apertura comercial de la ciudad al exterior sobre la actitud estaría operando en el sentido opuesto al hipotetizado, ya que sería mayor en la universidad privada de Monterrey. En segundo lugar, podría deberse a que los valores no son realmente más conservadores entre los estudiantes de la institución privada o la propia institución que 
entre los estudiantes de la institución pública o la propia institución. Queda sin posibilidad de contraste, con los presentes datos, el supuesto de los valores más conservadores en estudiantes de la institución privada y/o de la propia institución. Debe señalarse que la institución educativa privada no estaba financiada o ligada a ninguna orden religiosa, como sí ocurría en el estudio de Pulido et al. (2012), lo cual pudo motivar que las diferencias de valores no estuvieran cabalmente definidas entre la universidad pública y privada.

En relación al cuarto objetivo, la parcialización del sexo no alteró la significación de la diferencia de medias en ATG-S ni en el factor de rechazo sutil/interno, pero sí en EXT, y mostró que su efecto era difusor de la varianza en INT; por lo tanto, las diferencias aparecen en aspectos sutiles (ATG-S e INT) y no pueden ser atribuidas al sexo, esto es, son sustantivas, pero la diferencia en el aspecto manifiesto (EXT) finalmente es espuria y atribuible totalmente al sexo. En efecto, el componente de rechazo sutil/ interno fue el diferencial, donde se incluyen ATG-S e INT, pero no así el componente de rechazo abierto/externo, en el cual se incluye EXT. Mayor rechazo se asocia con el sexo masculino por la propia actitud cultural que repudia más la orientación no heterosexual entre hombres (Herek \& MacLemore, 2013), tal efecto del sexo explica la diferencia en el factor de manifestación pública de la diversidad sexual (EXT).

Con base en dichos datos, se puede afirmar que la carrera estudiada en la presente investigación sí tiene efecto sobre el rechazo sutil, pues los estudiantes de psicología muestran mayor aceptación, lo cual seguramente se deba a diferencias en sus planes de estudios y diferencias vocacionales, pero no a la mayor proporción de sexo femenino ni al hecho de que la facultad de psicología sea pública. Los estudiantes de psicología reciben educación sexual y son capacitados para impartirla, además, al profesional de psicología se le forma para atender trastornos sexuales, entre los cuales se encuentra la disforia con la orientación sexual o con el género; el objetivo de estos tratamientos es lograr una identidad positiva y la vivencia de una sexualidad integrada.

El presente estudio tiene varias limitaciones. Se reclutó una muestra no probabilística de estudiantes de medicina y psicología de universidades del noreste de México, así, las conclusiones derivadas de estos datos deben considerarse como hipótesis en esta población y otras afines. Los presentes datos corresponden a auto-reporte, por consiguiente, pudiesen diferir de los obtenidos con entrevistas, pruebas proyectivas o tiempos de reacción.

En conclusión, la actitud promedio en estos estudiantes de ciencias de la salud es de desacuerdo con rechazo abierto hacia las personas no heterosexuales, pero es ambigua hacia aspectos sutiles del rechazo (rango social, marginación en algunos derechos sociales y de familia) y hacia sentimientos homosexuales propios, en concordancia con el cambio de actitud en la sociedad occidental y la ideología 
heterosexista dominante. Existen diferencias entre las tres facultades en las subescalas ATG-S e INT y en el componente de rechazo sutil/interno, las cuales son sustantivas $y$ no atribuibles al sexo. También se observó diferencia de medias en la manifestación pública de la homosexualidad (EXT) entre las tres facultades, aunque, finalmente, la diferencia en este aspecto es engañosa o muy débil. En relación con estas tres subescalas (ATG-S, INT y EXT) y el componente de rechazo sutil/interno, el rechazo promedio fue estadísticamente equivalente entre los estudiantes de medicina y significativamente menor en estudiantes de psicología. Se puede afirmar que la carrera tiene un efecto significativo, tienen mayor aceptación las personas no heterosexuales entre estudiantes de psicología, probablemente por diferencias en los contenidos de su formación y aspectos vocacionales. La expectativa respecto a una media de rechazo más alto en los estudiantes de medicina de la universidad privada que en los estudiantes de medicina de la universidad pública no se cumplió; debido a que no se evaluaron los valores morales ni religiosidad y no se tenían datos sobre facultades de psicología y medicina públicas o privadas en las dos ciudades.El estudio no puede aclarar el posible efecto de la ciudad en el incumplimiento de la expectativa.

A la hora de evaluar el rechazo hacia las personas no heterosexuales se recomienda medir tanto aspectos abiertos como sutiles. Además, se sugiere evaluar heterosexismo y estudiar su asociación con el rechazo sutil y manifiesto. Más allá de la evaluación, se recomienda realizar talleres de cambio de actitud hacia aceptación de la diversidad sexual para crear conciencia de las manifestaciones sutiles de rechazo entre estudiantes de ciencias de la salud, especialmente entre estudiantes de medicina, y de este modo hacer efectivos los derechos de salud que poseen las personas no heterosexuales (CONAPRED, 2011). Un posicionamiento ambiguo entre la aceptación y el rechazo es la base desde la cual opera y persiste el rechazo sutil, de ahí que la intervención sea aconsejable. En los talleres para los estudiantes de ciencias de la salud se podrían trabajar como técnicas de investigación cualitativas, pruebas de asociación libre y entrevistas abiertas a personas no heterosexuales. Al analizar los datos sería importante hacer hincapié en el sesgo introducido por el grupo de identidad sexual y la ideología heterosexista. Podría invitarse a los talleres a personas no heterosexuales para generar contactos más próximos que faciliten el cambio de representación y actitud, asimismo, se podrían entablar debates de posicionamientos religiosos desde una ética de respeto hacia la diversidad (Moral \& Valle, 2013c; Whitehead \& Baker, 2012). 


\section{Referencias}

Barrientos, J., \& Cárdenas, M. (2013). Homofobia y calidad de vida de gays y lesbianas: Una mirada psicosocial. Psykhe, 22(1), 3-14. Burn, S. M., Kadlec, K., \& Rexer, R. (2005). Effects of subtle heterosexism on gays, lesbians, bisexuals. Journal of Homosexuality, 49(2), 23-38. doi:10.1300/J082v49n02_02

Campo, A., Díaz, A. J., \& Herazo, E. (2008). Homofobia en estudiantes de odontología e higiene oral: Revisión sistemática de la última década. Revista CES Odontología, 21(2), 63-68.

Campo, A., Herazo, E., \& Cogollo, Z. (2010). Homophobia in nursing students. Revista da Escola de Enfermagem da USP, 44(3), 839843.

Campo, A., \& Herazo, E. (2008). Homofobia en estudiantes de medicina: Una revisión de los diez últimos años. MedUNAB, 11(2), 120123.

Cárdenas, M., \& Barrientos, J. (2008). Actitudes explícitas e implícitas hacia los hombres homosexuales en una muestra de estudiantes universitarios en Chile. Psykhe, 17(2), 17-25. doi:10.4067/S0718-22282008000200002

Consejo Nacional para la Prevenir la Discriminación [CONAPRED]. (2011). Encuesta nacional sobre discriminación en México 2010. Resultados generales. México: CONAPRED.

Crompton, L. (2006). Homosexuality and civilization. Cambridge: Belknap.
Currie, M. R., Cunningham, E. G., \& Findlay, B. M. (2004). The Short Internalized Homonegativity Scale: Examination of the factorial structure of a new measure of internalized homophobia. Educational and Psychological Measurement, 64(6), 1053-1067. doi:10.1177/0013164404264845

Ellis, P. D. (2010). The essential guide to effect sizes: An introduction to statistical power, meta-analysis and the interpretation of research results. New York: Cambridge University Press.

Haddock, G. (2004). Contemporary perspectives on the psychology of attitudes. New York: Taylor \& Francis.

Herek, G. M. (1984). Attitudes toward lesbians and gay men: A factor analytic study. Journal of Homosexuality, 10(1/2), 39-51. doi:10.1300/J082v10n01_03

Herek, G. M. (2004). Beyond "homophobia": Thinking about sexual prejudice and stigma in the twenty-first century. Sexuality Research \& Social Policy, 1(2), 6-24. doi:10.1525/ srsp.2004.1.2.6

Herek, G. M., Gillis, J. R., \& Cogan, J. C. (2009). Internalized stigma among sexual minority adults: Insights from a social psychological perspective. Journal of Counseling Psychology, 56(1), 32-43. doi:10.1037/a0014672

Herek, G. M., \& McLemore, K. A. (2011). The Attitudes Toward Lesbians and Gay Men (ATLG) Scale. En T. Fisher, C. M. Davis, W. L. Yarber \& S. L. Davis (Eds.), Handbook of 
sexuality-related measures (3rd ed., pp. 415417). Oxford, England: Taylor \& Francis.

Herek, G. M., \& McLemore, K. A. (2013). Sexual prejudice. Annual Review of Psychology, 64, 309-333. doi:10.1146/annurevpsych-113011-143826

Hsueh-Hao, H. (2009). Homosexual behavior in the United States, 1988-2004: Quantitative empirical support for the social construction theory of sexuality. Electronic Journal of Human Sexuality, 12. Recuperado de http:// www.ejhs.org/Volume12/Homosexuality. htm

Klamen, D. L., Grossman, L. S., \& Kopacz, D. R. (1999). Medical student homophobia. Journal of Homosexuality, 37, 53-63. doi:10.1300/J082v37n01_04

Majied, K. (2008). A conceptual analysis of homophobia and heterosexism: Experiences of lesbian, gay, bisexual, transgendered and questioning people (LBGTQ) in Trinidad. The Caribbean Journal of Social Work, 6 \& 7, 144-166.

Matharu, K., Kravitz, R. L., McMahon, G. T., Wilson, M. D., \& Fitzgerald, F. T. (2012). Medical students' attitudes toward gay men. BMC Medical Education, 12, 71-78. doi:10.1186/1472-6920-12-71

Mayer, K. H., Bekker, L. G., Stall, R., Grulich, A. E., Colfax, G., \& Lama, J. R. (2012). Comprehensive clinical care for men who have sex with men: An integrated approach. Lancet, 380(9839), 378-387. doi:10.1016/ S0140-6736(12)60835-6
Moral, J., \& Martínez-Sulvarán, J. O. (2012). Validación de la Escala de Actitud hacia la Homosexualidad (EAH-10). Revista de Psicología Social, 27(2), 183-97.

Moral, J., \& Valle, A. (2011). Escala de Actitudes hacia Lesbianas y Hombres Homosexuales en México 1. Estructura factorial y consistencia interna. Revista Electrónica Nova Scientia, 3(2), 139-157.

Moral, J., \& Valle, A. (2012). Escala de Actitudes hacia Lesbianas y Hombres Homosexuales (ATLG) 2. Distribución y evidencias de validez. Revista Electrónica Nova Scientia, 4(1), 153-171.

Moral, J., \& Valle, A. (2013a). Dimensionalidad, consistencia interna y distribución de la Escala Homonegatividad Internalizada en estudiantes mexicanos de ciencias de la salud. Acta Mexicana de Psicología, 3(1), 22-50.

Moral, J., \& Valle, A. (2013b). Propiedades psicométricas de la escala de evaluación de la homofobia externalizada en estudiantes de ciencias de la salud mexicanos. Salud y Sociedad, 4(3), 230-247.

Moral, J., \& Valle, A. (2013c). Description and prediction of rejection toward gay men and lesbians among students of health sciences from Mexico. International Journal of Psychology Research, 8(4), 14-26.

Morrison, T. G., Speakman, C., \& Ryan, T. A. (2009). Irish university students' support for the human rights of gay men and lesbian women. Journal of Homosexuality, 56, 387400. doi:10.1080/00918360902728871 
Pulido, M. A., Huerta, A., Muñoz, F., Pahua, E., Pérez-Palacios, P., \& Saracho, S. (2012). Homofobia en universidades de México. Revista Internacional de Psicología y Educación, 15(2), 93-114.

Selleger, V. J., Bonke, B., \& Leeman, Y. A. M. (2006). Student diversity at Erasmus Medical Centre Rotterdam: Does it make any difference? Medical Teacher, 28(5), 142-148. doi:10.1080/01421590600776552

Sociedad Mexicana de Psicología (2007). Código ético del psicólogo (4 $4^{\mathrm{a}}$ ed.). México: Trillas.

Szymanski, D. M. (2008). Internalized heterosexism. Measurement, psychosocial correlates and research directions. Counseling Psychologist, 36, 525-574. doi:10.1177/0011000007309489

Whitehead, A. L., \& Baker, J. O. (2012). Homosexuality, religion, and science: Moral authority and the persistence of negative attitudes. Sociological Inquiry, 82(4), 487-509. doi:10.1111/j.1475-682X.2012.00425.X

Wienke, C., \& Gretchen, J. H. (2013). Does place of residence matter? Rural-urban differences and the wellbeing of gay men and lesbians. Journal of Homosexuality, 60(9), 1256-1279. doi:10.1080/00918369.2013.806166 